\title{
Lower risk of dementia with pioglitazone, compared with other second-line treatments, in metformin-based dual therapy: a population-based longitudinal study
}

\author{
Chieh-Hsiang Lu ${ }^{1,2,3} \cdot$ Chen-Yi Yang ${ }^{4}$ - Chung-Yi Li ${ }^{5,6}$ • Cheng-Yang Hsieh ${ }^{7}$. \\ Huang-Tz Ou ${ }^{4,8,9}$
}

Received: 11 August 2017 / Accepted: 13 October 2017 /Published online: 14 November 2017

(C) Springer-Verlag GmbH Germany, part of Springer Nature 2017

\begin{abstract}
Aims/hypothesis The effect of pioglitazone was compared with that of other second-line glucose-lowering drugs on the risk of dementia among individuals with type 2 diabetes receiving metformin-based dual therapy.

Methods A total of 204,323 individuals with type 2 diabetes aged $\geq 18$ years who were stable metformin users and dementia-free before the initiation of second-line glucose-lowering medication were identified in the period 2000-2011 from Taiwan's National Health Insurance Research Database and followed to the end of 2013. Primary analyses included 51,415 individuals aged $\geq 65$ years without dementia events in the first year of second-line glucose-lowering treatment. Study subjects were classified into mutually exclusive groups according to various second-line glucose-lowering drugs to metformin. Cox proportional hazards models were applied to assess the time-to-event between propensity scorematched glucose-lowering treatment groups.
\end{abstract}

Electronic supplementary material The online version of this article (https://doi.org/10.1007/s00125-017-4499-5) contains peer-reviewed but unedited supplementary material, which is available to authorised users.

Huang-Tz Ou

huangtz@mail.ncku.edu.tw

1 Division of Endocrinology and Metabolism, Department of Internal Medicine, Ditmanson Medical Foundation Chiayi Christian Hospital, Chiayi City, Taiwan

2 College of Chinese Medicine, China Medical University, Taichung, Taiwan

3 Department of Biotechnology, Asia University, Taichung, Taiwan

4 Institute of Clinical Pharmacy and Pharmaceutical Sciences, College of Medicine, National Cheng Kung University, 1 University Road, Tainan 7010, Taiwan
Results Individuals aged $\geq 65$ years on metformin + pioglitazone had a significantly lower risk of dementia compared with those on metformin + sulfonylurea (HR 0.56 ; $95 \%$ CI 0.34 , 0.93), and a lower, but insignificant, risk of dementia compared with those on other metformin-based dual regimens (i.e. metformin + acarbose, metformin + meglitinide, metformin + insulin or metformin + dipeptidyl peptidase 4 inhibitors). Among individuals aged $\geq 18$ years, there was also a decreased risk of dementia in those taking pioglitazone compared with those taking other second-line glucose-lowering drugs. A lower incidence of dementia was found in users of metformin + pioglitazone compared with users of metformin + rosiglitazone.

Conclusions/interpretation Pioglitazone as a second-line treatment after metformin might provide a protective effect on dementia risk among individuals with type 2 diabetes.

Keywords Dementia · Glucose-lowering drugs · Pioglitazone $\cdot$ Type 2 diabetes mellitus

5 Department of Public Health, College of Medicine, National Cheng Kung University, Tainan, Taiwan

6 Department of Public Health, China Medical University, Taichung, Taiwan

7 Department of Neurology, Tainan Sin Lau Hospital, Tainan, Taiwan

8 Department of Pharmacy, College of Medicine, National Cheng Kung University, Tainan, Taiwan

9 Department of Pharmacy, National Cheng Kung University Hospital, Tainan, Taiwan 


\begin{tabular}{|c|c|}
\hline \multicolumn{2}{|c|}{ Abbreviations } \\
\hline CIC & Catastrophic illness certificate \\
\hline DPP-4 & Dipeptidyl peptidase 4 \\
\hline ITT & Intention-to-treat \\
\hline LHDB & $\begin{array}{l}\text { Longitudinal Cohort of Diabetes } \\
\text { Patients Database }\end{array}$ \\
\hline NHI & National Health Insurance \\
\hline NHIRD & National Health Insurance Research Database \\
\hline PPAR- $\gamma$ & $\begin{array}{l}\text { Peroxisome proliferator-activated } \\
\text { receptor gamma }\end{array}$ \\
\hline TZDs & Thiazolidinediones \\
\hline
\end{tabular}

\section{Introduction}

The established association between diabetes and dementia risk [1-6] has raised interest in the role of glucose-lowering drugs in the prevention of dementia. Thiazolidinediones (TZDs) are potent insulin-sensitisers. Dementia is characterised by impaired glucose utilisation in the brain and a glial-mediated inflammatory response, which suggests a potential role of TZDs in the treatment of dementia [7-9].

Several observational studies $[2,4,10]$ have evaluated the association between the use of TZDs and the risk of dementia, but the results are inconclusive and should be interpreted with caution given the relatively small sample sizes in TZD studies $(n=28)$ [4]; follow-up periods that were too short to catch chronic conditions such as dementia; serious concern regarding confounding by indications that arise when only comparing glucose-lowering treatment with non-treatment (i.e. metformin vs no metformin $[2,10]$, sulfonylureas vs no sulfonylureas [10], TZDs vs no TZDs [2, 10], insulin vs no insulin [2, 10], pioglitazone vs no pioglitazone [2]); and the threat of selection bias due to the nature of observational designs [11]. No studies have assessed the comparative dementia risk of different glucose-lowering drugs based on the design of active comparator and new users of treatment, to mitigate potential biases (e.g. confounding by indication). Dipeptidyl peptidase 4 (DPP-4) inhibitors, a new class of glucoselowering drugs, have not been evaluated in terms of their association with the risk of dementia in diabetic individuals.

Against this background, the present study investigated a large nationwide longitudinal cohort of incident type 2 diabetes cases followed for up to 14 years to evaluate the comparative risks of developing dementia associated with different glucose-lowering drugs. Because metformin is recommended in recent guidelines as the first-line treatment [12], and most individuals with type 2 diabetes in Taiwan today are prescribed metformin [13], we focused on metformin-based dual regimens to compare the effects of second-line treatments. Among second-line glucose-lowering drugs, we hypothesised that pioglitazone exerts a more protective effect on dementia risk compared with other second-line glucose-lowering drugs.
A large cohort study showed that pioglitazone was the most promising glucose-lowering treatment to reduce dementia risk [2]; however, the study only compared exposure to pioglitazone with non-exposure, rather than with exposure to other glucose-lowering drugs. To test our hypothesis, we therefore designed active treatment comparisons in which pioglitazone served as a comparator for other glucose-lowering drugs.

\section{Methods}

The Institutional Review Board of National Cheng Kung University Hospital approved the study before commencement (A-ER-103-298).

\section{Data source}

The Longitudinal Cohort of Diabetes Patients Database (LHDB) 1996-2013 was the main dataset used in this study. The LHDB is the most representative longitudinal cohort of diabetic individuals retrieved from the National Health Insurance Research Database (NHIRD) provided by Taiwan's National Health Insurance (NHI), which is a mandatory enrolment, single-payment system that covers over 99\% of Taiwan's population. The LHDB includes a random sample of 120,000 de-identified incident diabetes cases from each calendar year, tracked back to 1996 and followed to 2013 to establish a longitudinal cohort. The application of the LHDB is described elsewhere [13-15]. Since the LHDB is anonymised, informed consent from study participants was not required by the Institutional Review Board.

\section{Study cohort}

We selected individuals aged $\geq 18$ years at diagnosis of type 2 diabetes (ICD-9-CM: 250.0X-250.9X, X=0 or 2; www. icd9data.com/2007/volume1) between 1999 and 2013 in the LHDB. We excluded cases with unknown date of birth or sex $(n=247)$. We identified metformin-based dual regimen users between 2000 and 2011. The date at which a second-line glucose-lowering drug (i.e. a sulfonylurea, pioglitazone, meglitinide, acarbose or insulin) was first added to metformin served as the index date. Those who had taken any second-line glucose-lowering drugs before the index date were excluded in order to include only new users of second-line glucoselowering drugs (i.e. those who were naïve to second-line glucose-lowering treatments in the 180 days prior to the index date). Previous diabetes studies have considered 180 days to be a sufficient washout period $[14,15]$ to define new users/ initiators of glucose-lowering treatments. Also, we only selected stable users of second-line glucose-lowering drugs, who were defined as those with at least three consecutive refills of second-line glucose-lowering medication after the 
index date and with any gaps between two consecutive refills of fewer than 30 days (grace period) $[14,15]$. We excluded those with dementia before the index date, in order to ensure that the dementia cases diagnosed after the index date were incident dementia. A total of 204,323 individuals with type 2 diabetes aged $\geq 18$ years newly using metformin-based therapies were identified, of which 52,006 individuals aged $\geq 65$ years were included in our primary analyses (Fig. 1). Cases with dementia events that occurred within 1 year after the index date (i.e. immediate events after the start of treatment) were excluded from our primary analyses. Our main analyses therefore included 51,415 subjects aged $\geq 65$ years without dementia before the initiation or during the first year of second-line glucose-lowering treatment.

\section{Study outcome}

Incident events of dementia were identified from both outpatient and inpatient LHDB files according to the presence of at least two outpatient records or one inpatient record of a diagnosis of dementia (A codes A210, A222; or ICD-9-CM disease diagnosis codes 290.0, 290.1, 294.1, 331.0-331.2, 331.7-331.9) within any 1 year of follow-up [4]. These diagnosis codes are commonly used in the studies using the NHIRD to identify diabetic individuals with dementia $[4,16]$. The observation period for each case was from the index date to the date of death or the end of 2013. The maximum follow-up period was 14 years (2000-2013) and the minimum was 2 years (2012-2013). The minimum follow-up period (i.e. 2 years) is supported by a previous study [17].

\section{Exposure to glucose-lowering drugs}

Medication use was identified using 'drug_no' codes in the NHIRD and linked to the Anatomical Therapeutic Chemical Classification System used to classify active ingredients of glucose-lowering drugs, namely metformin, sulfonylureas, DPP-4 inhibitors, pioglitazone, meglitinides, acarbose and insulin. Study subjects, all on metformin-based dual regimens, were further classified into five mutually exclusive groups according to second-line treatment at the index date (i.e. the date the second-line treatment was added to metformin): metformin + sulfonylurea, metformin + pioglitazone, metformin + meglitinide, metformin + acarbose and metformin + insulin. We used an active treatment comparison design in which pioglitazone served as a comparator for other glucose-lowering drugs. We applied propensity score matching to mitigate potential bias due to lack of distribution overlap and due to different density weight when conducting an observational study [18]. We used propensity score matching by the nearest neighbour approach without replacement within a calliper of 0.025 on the propensity scores at a fixed ratio of $1: 1$ [19] to match metformin + pioglitazone with another metformin- based regimen (e.g. metformin + pioglitazone vs metformin + sulfonylurea). We estimated the propensity scores by using a logistic regression model, where treatment status (e.g. metformin + pioglitazone vs metformin + sulfonylurea) was a dependent variable and a list of independent variables (Table 1) were considered to be associated with both the selection of glucose-lowering drugs and the risk of developing dementia, including demographics (e.g. age), comorbidity history (measured by the Charlson Comorbidity Index [20]), diabetic complications (measured using the adapted Diabetes Complication Severity Index [21]), diabetes duration (i.e. the time from diabetes onset to the date of initiation of second-line glucose-lowering treatment), cumulative metformin exposure (in terms of defined daily dose) from metformin initiation to addition of a second-line glucose-lowering drug, cardiovascular disease history from 1 year before the index date (e.g. stroke), and medication history for cardiovascular diseases (e.g. $\beta$-blockers, angiotensin receptor blockers).

\section{Statistics}

Descriptive analyses Subject characteristics were analysed using descriptive statistics, including means and standard deviations for continuous variables, and frequency and proportion for dichotomous or categorical variables. The crude incidence rate of dementia events was calculated as the total number of events during the follow-up period divided by the number of person-years at risk. The number of person-years at risk was defined as the sum of study subjects from the index date (the first claim of a second-line glucose-lowering prescription) to the diagnosis of the first event, death or the end of 2013, whichever came first, in the intention-to-treat (ITT) scenario.

Analytic analyses Our primary analyses targeted individuals aged $\geq 65$ years without dementia before the index date and within 1 year after the index date. Our secondary analyses examined the cohorts of individuals aged $\geq 18$ years. Cox proportional hazards regression was applied to evaluate the timeto-event for the two matched groups. The $\log (-\log$ (survival)) vs log of survival time graph (using the PROC LIFETEST function in SAS, version 9.4; SAS Institute, Cary, NC, USA) resulted in parallel curves, implying that the proportional hazard assumption of the Cox model in this study was satisfied [22]. HRs and their 95\% CIs were computed. The significance level of this study was set at 0.05 .

Sensitivity analyses First, in order to include DPP-4 inhibitors, we changed the case identification period from 2000 2011 to 2009-2011 and then re-analysed the data using the previously mentioned approaches. A total of 59,693 individuals with type 2 diabetes aged $\geq 18$ years newly on metforminbased therapies in 2009-2011 were included (Fig. 1). Second, we used the as-treated scenario, where study subjects were 
Cases from 1999-2011 LHDB $(n=632,528)$

- Type 2 diabetes (ICD-9-CM: 250.0X-250.9X, X=0 or 2 ) and aged $>18$ years

- Stable metformin users (three consecutive refills and any gaps between two refills $<30$ days)

\section{$\downarrow$}

New users of metformin-based dual therapy ( $n=204,323)$

- Second-line glucose-lowering drug was added during 2000-2011 (first date of second-line add-on defined as index date), and stable users

- Exclude those who had second-line drug 180 days prior to index date

- Exclude those who did not have metformin 365 days prior to index date

- Exclude those diagnosed with dementia (A code: A210, A222; ICD-9-CM: 290.0, 290.1, 294.1, 331.0-331.2, 331.7-331.9) before index date

\section{$\downarrow$}

Metformin-based dual therapy cohort

- Metformin + sulfonylurea $(n=181,798)$

- Metformin + acarbose $(n=8775)$

- Metformin + meglitinide $(n=9209)$

- Metformin + pioglitazone $(n=3330)$

- Metformin + insulin ( $n=1211)$

\section{$\downarrow$}

Patients $\geq 65$ years $(n=52,006)$

- Metformin + sulfonylurea $(n=44,951)$

- Metformin + acarbose $(n=2822)$

- Metformin + meglitinide $(n=3122)$

- Metformin + pioglitazone $(n=850)$

- Metformin + insulin $(n=261)$

\section{$\downarrow$}

Sensitivity analysis to change case identification period from 2000-2011 to 2009-2013 to include DPP-4

inhibitors

New users of metformin-based dual therapy $(n=59,693)$

- Second-line glucose-lowering drug was added during 2009-2011 (first date of second-line add-on defined as index date), and stable users

- Exclude those who had second-line drug 180 days prior to index date

- $\quad$ Exclude those who did not have metformin 365 days prior to index date

- Exclude those diagnosed with dementia (A code: A210, A222; ICD-9-CM: 290.0, 290.1, 294.1, 331.0$331.2,331.7-331.9)$ before index date

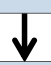

Metformin-based dual therapy cohort

- Metformin + sulfonylurea $(n=43,709)$

- Metformin + acarbose $(n=4462)$

- Metformin + meglitinide $(n=3237)$

- Metformin + pioglitazone $(n=2067)$

- Metformin + DPP-4 inhibitor $(n=4867)$

- Metformin + insulin $(n=1351)$

\section{$\downarrow$}

Patients $\geq 65$ years $(n=17,963)$

- Metformin + sulfonylurea $(n=12,451)$

- Metformin + acarbose $(n=1654)$

- Metformin + meglitinide $(n=1460)$

- Metformin + pioglitazone $(n=587)$

- Metformin + DPP-4 inhibitor $(n=1483)$

- Metformin + insulin $(n=328)$

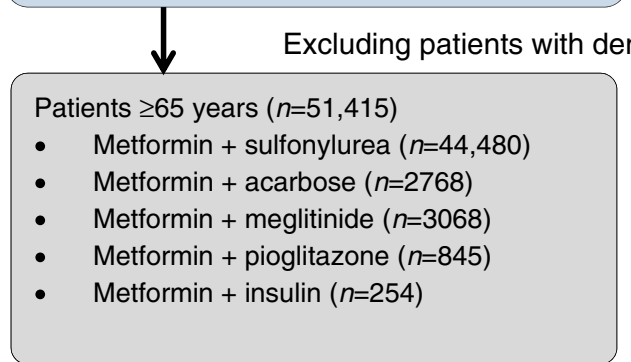

Fig. 1 Flow diagram of study population selection

censored when their original dual glucose-lowering treatment changed (i.e. switched to another first- or second-line glucoselowering drug, added a third-line glucose-lowering drug, or discontinued metformin or second-line glucose-lowering treatment), in addition to the censored points in the analyses based on the ITT scenario (i.e. lost to follow-up, death or end of 2013). Third, our primary analyses did not include rosiglitazone because its use significantly dropped in Taiwan during 2008-2013 [23] due to an elevated risk of heart attacks $[24,25]$. In the sensitivity analyses, we combined both pioglitazone and rosiglitazone into the TZD category as comparator to other second-line glucose-lowering drugs and then re- 
analysed the data. Also, we compared pioglitazone and rosiglitazone individually in terms of their effects on dementia incidence. Fourth, we tested interaction effects of age and sex with pioglitazone, and performed age- (i.e. age 18-64 years vs age $\geq 65$ years) and sex-stratified analyses. Fifth, in the ITT scenario, as we did not control for the pattern of metformin during follow-up (i.e. from index date to censored point), we further conducted analyses which adjusted for the yearly dosage of metformin (in terms of defined daily dose) in the Cox models under the ITT scenario.

\section{Results}

Our primary analyses were based on the cohort aged $\geq 65$ years identified in the period 2000-2011. The characteristics of individuals in two treatment groups are presented in Table 1. After propensity score matching, the patients on metformin + sulfonylurea had more heart failure events before the index date. The patient characteristics of the cohort aged $\geq 65$ years extracted from the 2009 2011 data are presented in Table 2. Table 3 shows that individuals $\geq 65$ years taking metformin + pioglitazone had a lower incidence rate of dementia and a significantly lower risk of dementia compared with matched individuals taking metformin + sulfonylurea (adjusted HR 0.56, [95\% CI: $0.34,0.93])$. The metformin + pioglitazone group also had a lower, though not significantly lower, risk of dementia compared with all other metformin dual regimens (adjusted HR [95\% CI]: 0.66 [0.39, 1.12] for metformin + pioglitazone vs metformin + acarbose, 0.69 $[0.41,1.16]$ for metformin + pioglitazone vs metformin + meglitinide, $0.98[0.42,2.25]$ for metformin + pioglitazone vs metformin + insulin). The results for the ITT and as-treated scenarios consistently indicated lower incidence rates of dementia in those who used metformin + pioglitazone compared with those using other metforminbased dual regimens (Table 3). The results of the analyses of the 2009-2011 cohort are similar to those of the 2000 2011 cohort (Table 4). Table 4 also shows that the users of metformin + pioglitazone had a lower, though not significantly lower, risk of dementia compared with those on metformin + DPP-4 inhibitors (adjusted HR 0.59 [95\% CI: $0.25,1.37])$. Because the number of dementia events in the analyses based on the as-treated scenario was relatively few in the 2009-2011 cohort, we did not conduct further analyses based on the as-treated scenario.

Electronic supplementary material (ESM) Tables 1 and 2 show the characteristics of individuals aged $\geq 18$ years identified in 2000-2011 and 2009-2011, respectively. ESM Tables 3 and 4 show the results of adjusted HRs and $95 \%$ CIs of individuals aged $\geq 18$ years identified in 2000-2011 and 2009-2011, respectively; the analyses only excluded dementia cases before the index date but did not exclude cases with dementia that occurred within 1 year after the index date. Although the relationship between the use of metformin + pioglitazone and the risk of dementia based on the analysis of subjects aged $\geq 18$ years (ESM Tables 3 and 4 ) is essentially consistent with that derived from the primary analyses of subjects aged $\geq 65$ years (Tables 3 and 4), the former analyses showed even more significant findings, probably due to a larger sample size in the cohort of individuals aged $\geq 18$ years.

When we included both rosiglitazone and pioglitazone in the category of TZDs, TZD users had a lower risk of dementia compared with users of other second-line glucose-lowering drugs (ESM Table 5). Also, we further constructed two propensity score-matched groups for metformin + pioglitazone $(n=1881)$ and metformin + rosiglitazone $(n=1881)$ and compared dementia risk between the groups. The incidence rates of dementia in the metformin + pioglitazone and metformin + rosiglitazone groups were, respectively, 2.38 and 3.89 per 1000 person-years based on the ITT scenario and 0.36 and 0.46 per 1000 person-years based on the as-treated scenario. A lower, but insignificant, dementia risk was associated with the use of metformin + pioglitazone vs metformin + rosiglitazone (adjusted HR 0.72, $p=0.2097$, based on the ITT scenario; adjusted HR 0.52, $p=0.6461$, based on the as-treated scenario). Furthermore, there was no significant interaction effect of age and sex. The protective effects of pioglitazone over other second-line glucose-lowering drugs were observed in age- and sex-stratified subgroups (ESM Table 6), although the number of dementia events was limited in the subgroup analyses, which comprised the power for the statistical testing. Finally, the results based on the Cox model analyses which adjusted for yearly metformin dosage in follow-up (ESM Table 7) were consistent with those from the analyses that did not adjust for yearly metformin dosage (ESM Table 3).

\section{Discussion}

This large diabetes cohort study (i.e. $n=204,323$ in the 2000 2011 cohort; Fig. 1) with long-term follow-up (median follow-up 5.59 and 4.97 years, respectively, for those aged $\geq 18$ years and $\geq 65$ years) provides empirical evidence of the comparative effects of pioglitazone vs other second-line glucose-lowering drugs on the risk of dementia among individuals with type 2 diabetes. The effects of TZDs (combined pioglitazone and rosiglitazone) on dementia risk have been investigated previously. Preclinical data suggest that longterm use of peroxisome proliferator-activated receptor gamma (PPAR- $\gamma$ ) agonists such as TZDs prevents the neuropathological and behavioural changes associated with different types of 


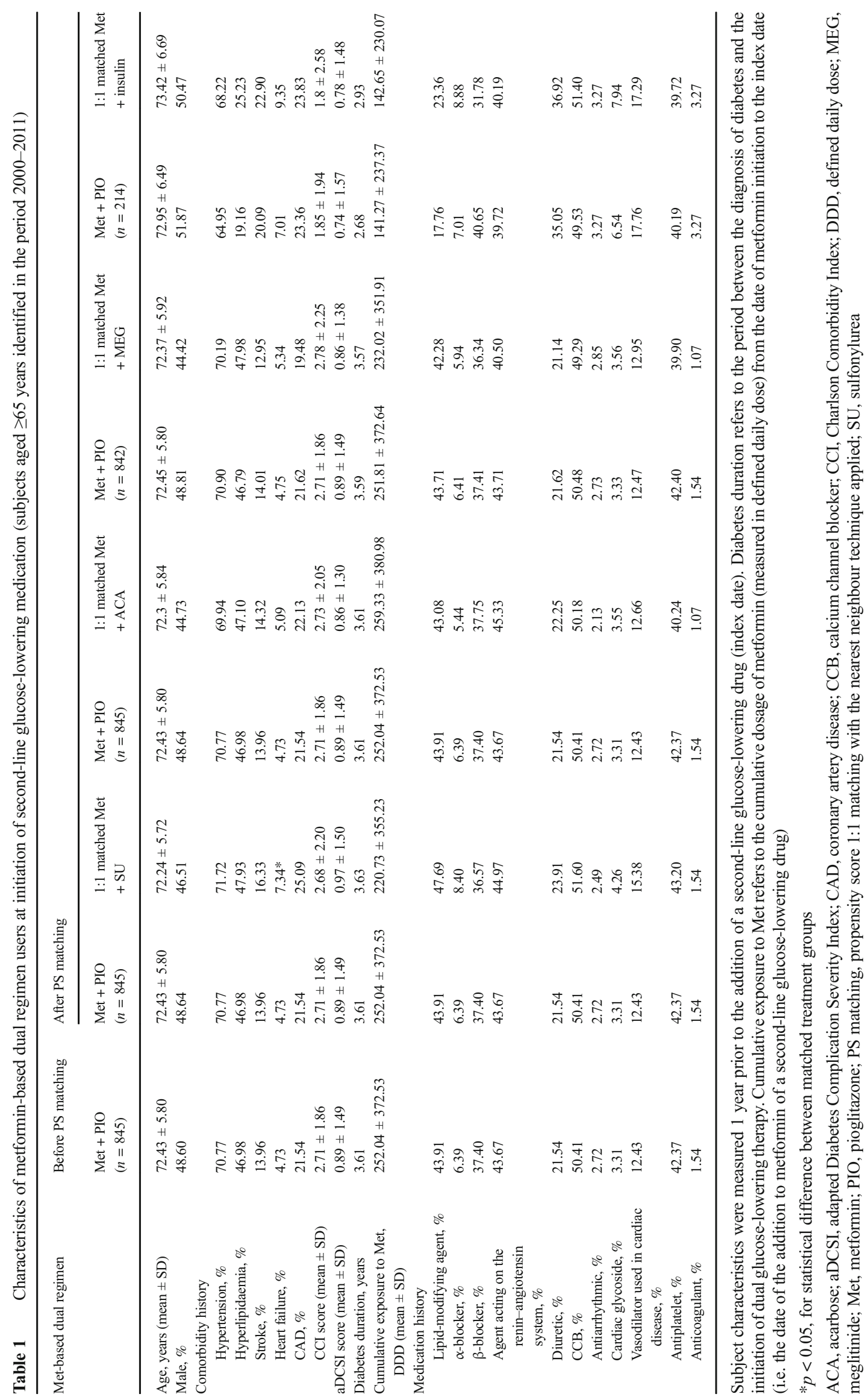




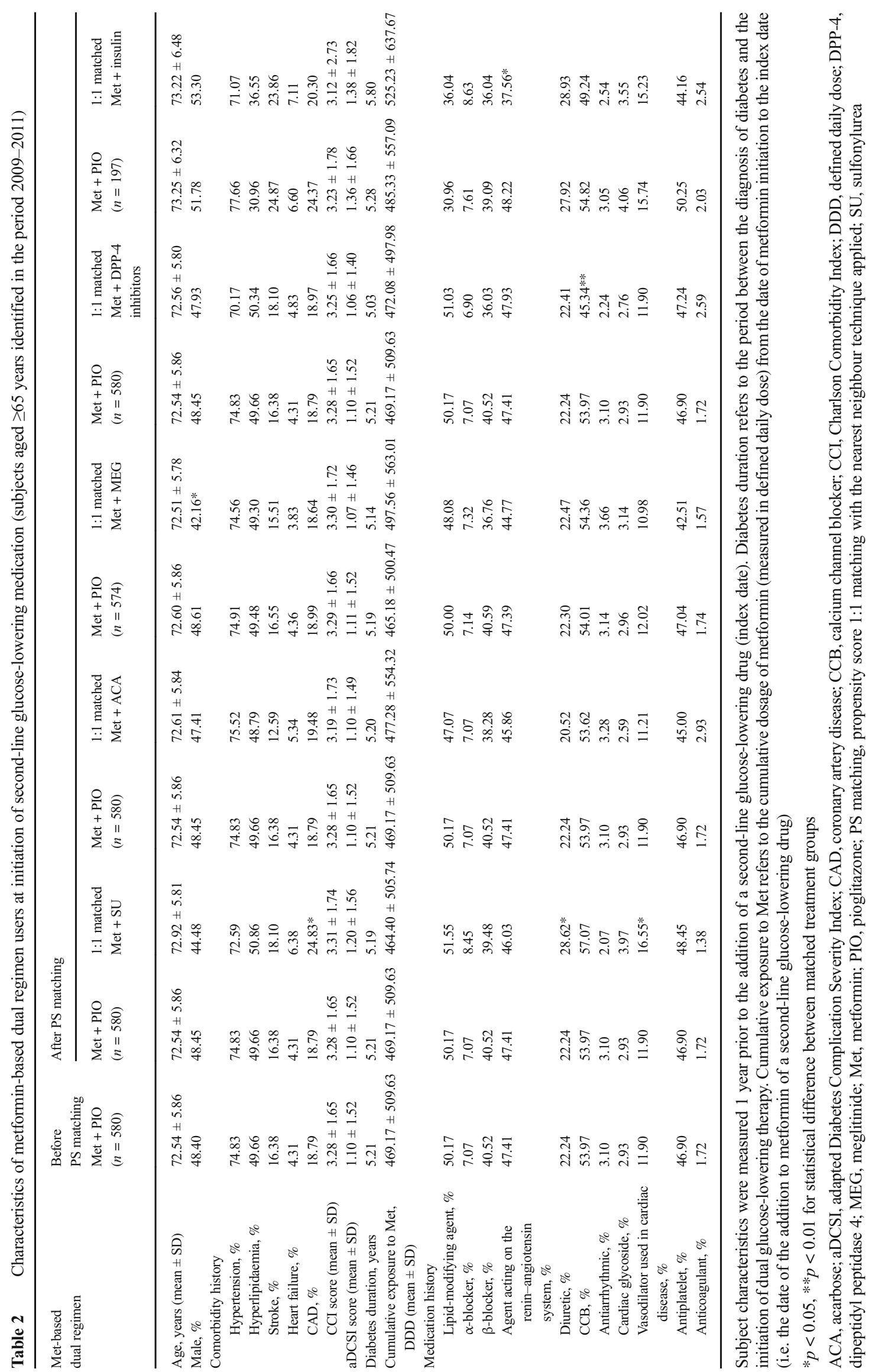




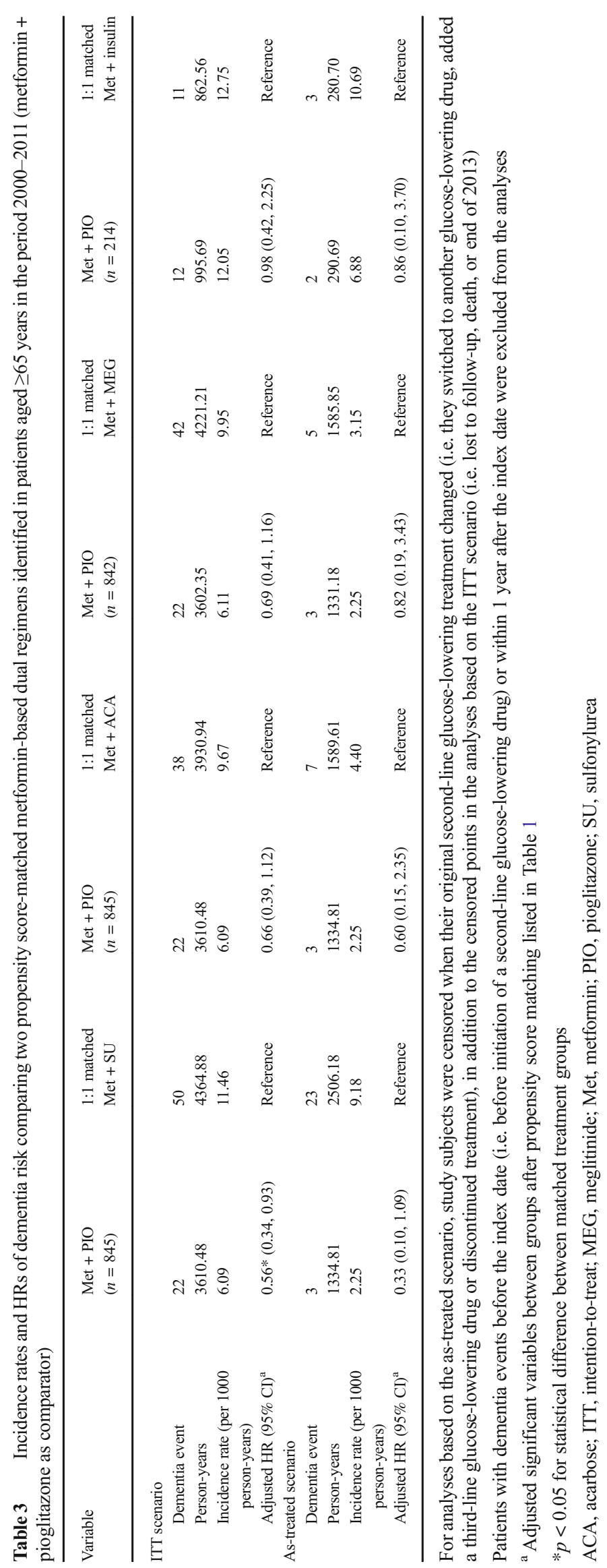




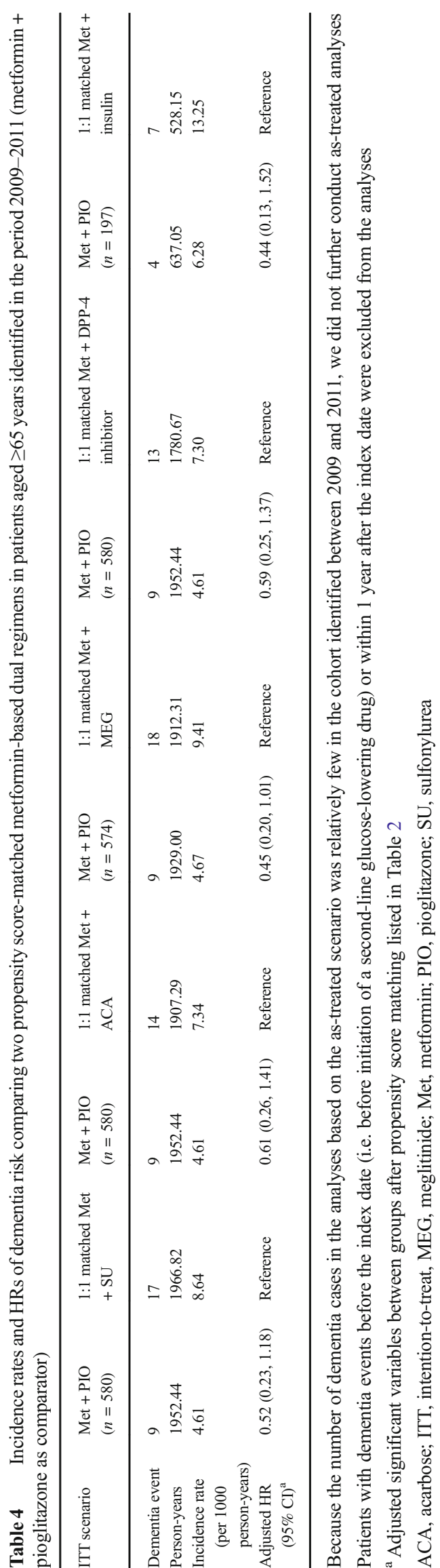


dementia (e.g. Alzheimer's disease and vascular dementia) [26]. Specifically, PPAR- $\gamma$ is expressed in the brain at low levels under physiological conditions and exhibits a range of activities that positively influence the pathology of Alzheimer's disease, including ameliorating the inflammatory status of the Alzheimer's disease brain by repressing the secretion of proinflammatory molecules, enhancing mitochondrial function and involving the processing of the amyloid $\beta$ peptide [27]. Also, a previous animal study showed that PPAR- $\gamma$ agonists significantly reversed diabetes-induced vascular dementia through their multiple actions, including antioxidative and anti-acetylcholinesterase activity and anti-inflammatory and neuroprotective actions [28]. In the present study, we found that patients taking TZDs had a lower risk of dementia compared with those taking other second-line glucose-lowering drugs (ESM Table 5). By contrast, Cheng et al found that sulfonylurea or metformin use conferred a lower dementia risk compared with TZDs, and that users of TZDs had a significantly higher risk of dementia compared with those who had never used TZDs [4]. However, the number of patients using TZDs in that study were relatively few $(n=28)$ [4], which may threaten the internal validity of the findings.

Pioglitazone, a TZD medication, could be a viable therapeutic agent for dementia, but it is still under investigation, especially in humans. Animal studies have shown that pioglitazone attenuates streptozotocin-induced memory deficits in mice [29] and improves cognitive impairment in 1-methyl-4phenyl-1,2,3,6-tetrahydropyridine (MPTP)-treated monkeys [30]. The first human research on the cognitive effects of pioglitazone showed that daily use of pioglitazone for 6 months among individuals with comorbid dementia and type 2 diabetes increased their logical memory [31] and improved cognitive performance [32] compared with individuals who did not receive pioglitazone. However, these studies included a small number of subjects (i.e. 15 pioglitazone users and 17 non-users [31]; 21 pioglitazone users and 21 non-users [32]) and assessed type 2 diabetes individuals with existing cognitive impairment. A recent large German cohort study of 145,928 individuals aged $\geq 60$ years who were initially free of dementia and diabetes found that pioglitazone use was associated with a reduced risk of dementia [2]. Longer pioglitazone use (i.e. eight or more calendar quarters) led to a lower dementia risk [2]. Compared with diabetic individuals not on pioglitazone, pioglitazone users had a $23 \%$ lower risk of dementia (RR 1.23, non-use vs use of pioglitazone; $p<0.001$ ) [2]. Notably, the present study extends existing knowledge on the cognitive effects of pioglitazone use vs non-use [2, 31, 32] by comparing the cognitive effects of pioglitazone use with those of various second-line glucose-lowering drugs.

The German study also found that rosiglitazone use vs nonuse led to a reduced risk of dementia in diabetic individuals $\geq 60$ years, but this cognitive protective effect did not reach the level of statistical significance and was lower than that of pioglitazone use (RR 0.84, rosiglitazone use vs non-use, $p=0.328$; RR 0.53, pioglitazone use vs non-use, $p=0.029$ ) [2]. Similarly, the present study found that patients receiving pioglitazone had a lower incidence of dementia compared with propensity score-matched rosiglitazone-treated patients, implying a better protective effect of pioglitazone over rosiglitazone.

The present study has the following strengths. First, it was a large nationwide population-based study highly representative of individuals with type 2 diabetes in Taiwan and it had a long follow-up period (2000-2013). Second, its design included active treatment comparisons (rather than comparing drug use with non-use). The study identified patients who were newly starting second-line glucose-lowering treatment, and excluded those who had a history of dementia before starting second-line glucose-lowering treatment as well as those who developed dementia in the first year of secondline glucose-lowering treatment. These efforts are expected to mitigate potential confounding by indication, as observed in previous studies $[2,10]$, and prevent the bias due to including immediate events that might not have been associated with glucose-lowering treatment. Third, propensity score matching was applied to achieve balance in subject baseline characteristics, thus reducing potential selection bias. Fourth, different identification periods for study cases (i.e. 2009-2011) were modelled to assess the cognitive effects of newer glucoselowering drugs (i.e. DPP-4 inhibitors), which verifies the validity of the study findings. Finally, considering nonadherence or low adherence to glucose-lowering drugs commonly observed in practice (i.e. discontinuing or switching to or adding another glucose-lowering drug), we applied the astreated scenario, where subjects were censored when the treatment regimen changed. The results from the as-treated scenario were consistent with our primary analyses based on the ITT scenario, which supports the validity of the study.

Several limitations should be addressed. First, some laboratory data (e.g. $\mathrm{HbA}_{1 \mathrm{c}}$ ) were not available in the NHIRD claims data. However, we used surrogate indicators to adjust for the diabetes severity of study subjects, including the adapted Diabetes Complication Severity Index, diabetes duration, and cumulative exposure to metformin before the index date (i.e. before the initiation of second-line glucose-lowering treatment). Second, we used 180 days as a washout period to define new users/ initiators of glucose-lowering treatments, based on clinical practice in Taiwan where diabetic individuals who are stable on glucose-lowering treatments usually return for follow-up visits every month, and the prescription refill period is unlikely to be longer than 3 months. Doctors must evaluate patients' clinical profiles (e.g. $\mathrm{HbA}_{1 \mathrm{c}}$ and fasting blood sugar) every 30-90 days and then adjust their glucose-lowering treatment regimens if needed. This means that, even if a patient receives the same 
glucose-lowering drug after a 90 day interval, s/he would be considered a new user of that particular drug. Although a washout period of 180 days is clinically and practically plausible, we were unable to check the entire history of medication use for each individual subject because the claims data available to this study can only be traced back to 1999 . Similarly, we used 365 days as a stable treatment period for glucose-lowering medication (e.g. metformin), which is approximately four times sequential 3 month glucose-lowering prescription refills (i.e. 360 days). However, we were also unable to check whether the subjects did indeed persistently take metformin based on the claims data we used.

There have been no validation studies on the ICD-9 diagnosis codes for the definition of dementia in Taiwan. Taiwan's NHI, however, issues a catastrophic illness certificate (CIC) for dementia, which is classified as 'senile and pre-senile organic psychotic conditions'. The CIC in Taiwan is typically considered to be valid in disease classification, because strict conditions have to be met before a CIC is issued (e.g. several clinical profile/ data documents are required) by Taiwan's NHI. Patients with a CIC do not have to pay for their medical care. However, a CIC application for dementia is strict: only neurologists or psychiatrists are allowed to submit a CIC application for dementia; furthermore, these patients usually also have other severe psychotic disorders. As a result, not all individuals with dementia in Taiwan have a CIC for dementia. We have tried to use both disease diagnosis codes (i.e. ICD-9 or A codes) and CIC approaches to classify dementia events. However, the number of cases of dementia confirmed by both disease diagnosis codes and CIC approaches (i.e. a patient having an ICD-9 code and also a CIC for dementia) was much smaller than that identified by disease diagnosis codes only (e.g. the number of dementia cases among metformin + pioglitazone users aged $\geq 18$ years was 1 vs 39 , respectively, according to disease diagnosis codes and a CIC or according to disease diagnosis codes only). This implies that only a few dementia cases can be identified by a CIC, and these individuals may not be representative of all those with dementia in Taiwan. This is why previous Taiwanese studies on dementia [4, 16, 33-35] only relied on disease diagnosis codes to capture dementia events when the NHIRD was used.

Although we had a long follow-up period in which to observe dementia events, we did not evaluate the long-term use of pioglitazone on dementia prevention. Treatment dose or duration or length of response to pioglitazone deserves future research. Moreover, the results of protective HRs for dementia when metformin + pioglitazone compared with other dual regimens from the as-treated analyses should be interpreted with caution due to the small number of dementia cases in the analyses. As such, the point estimates of HRs may be unduly influenced by the small number of event cases in the analysis, which can be further evidenced by wide CIs corresponding to these point estimates. Finally, the effect of glucose-lowering treatment may vary according to the type of dementia (e.g. Alzheimer's disease or vascular dementia). However, this study only treated dementia as a single entity.

\section{Conclusion}

In this real-world observational study, we found that the use of pioglitazone may be associated with a lower risk of dementia compared with other second-line glucose-lowering drugs added to metformin, with effects that are independent of age and sex. Future prospective trials are needed to verify a possible cognitive protective effect of pioglitazone.

Acknowledgements We gratefully thank National Cheng Kung University and its affiliated hospital for their support.

Data availability Data sharing is not applicable to this study as data management and analysis were performed on a statistics server through remote access in National Cheng Kung University in Taiwan, for privacy and safety concerns.

Funding This work was supported by a grant from the Ministry of Science and Technology, Taiwan (MOST 104-2320-B-006-008-MY3) and a grant from the Ditmanson Medical Foundation Chia-Yi Christian Hospital Research Programme (R105-012).

Duality of interest The authors declare that there is no duality of interest associated with this manuscript.

Contribution statement All authors contributed to the study design, acquisition of data and interpretation of results, as well as the drafting and critical revision of the article for important content. HTO is the guarantor of this work, has full access to all the data in the study, and takes responsibility for the integrity of the data and accuracy of the data analysis. The final version was approved by all authors.

\section{References}

1. Ohara T, Doi Y, Ninomiya T et al (2011) Glucose tolerance status and risk of dementia in the community: the Hisayama Study. Neurology 77:1126-1134

2. Heneka MT, Fink A, Doblhammer G (2015) Effect of pioglitazone medication on the incidence of dementia. Ann Neurol 78:284-294

3. Moore EM, Mander AG, Ames D et al (2013) Increased risk of cognitive impairment in patients with diabetes is associated with metformin. Diabetes Care 36:2981-2987

4. Cheng C, Lin C-H, Tsai Y-W, Tsai C-J, Chou P-H, Lan T-H (2014) Type 2 diabetes and antidiabetic medications in relation to dementia diagnosis. J Gerontol Ser A Biol Med Sci 69:1299-1305

5. Chatterjee S, Peters SA, Woodward M et al (2016) Type 2 diabetes as a risk factor for dementia in women compared with men: a pooled analysis of 2.3 million people comprising more than 100 , 000 cases of dementia. Diabetes Care 39:300-307 
6. Lu F-P, Lin K-P, Kuo H-K (2009) Diabetes and the risk of multisystem aging phenotypes: a systematic review and meta-analysis. PLoS One 4:e4144

7. Ghisletti S, Huang W, Ogawa S et al (2007) Parallel SUMOylationdependent pathways mediate gene-and signal-specific transrepression by LXRs and PPAR $\gamma$. Mol Cell 25:57-70

8. Straus DS, Glass CK (2007) Anti-inflammatory actions of PPAR ligands: new insights on cellular and molecular mechanisms. Trends Immunol 28:551-558

9. Craft S (2007) Insulin resistance and Alzheimer's disease pathogenesis: potential mechanisms and implications for treatment. Curr Alzheimer Res 4:147-152

10. Imfeld P, Bodmer M, Jick SS, Meier CR (2012) Metformin, other antidiabetic drugs, and risk of Alzheimer's disease: a populationbased case-control study. J Am Geriatr Soc 60:916-921

11. Lund JL, Richardson DB, Stürmer T (2015) The active comparator, new user study design in pharmacoepidemiology: historical foundations and contemporary application. Curr Epidemiol Rep 2:221228

12. American Diabetes Association (2017) 8. Pharmacologic approaches to glycemic treatment. Diabetes Care 40(Suppl 1):S64S74

13. Ou HT, Chang KC, Liu YM, Wu JS (2017) Recent trends in the use of antidiabetic medications from 2008 to 2013: a nation-wide population-based study from Taiwan. J Diabetes 9:256-266

14. Ou HT, Chang KC, Li CY, Wu JS (2016) Risks of cardiovascular diseases associated with dipeptidyl peptidase-4 inhibitors and other antidiabetic drugs in patients with type 2 diabetes: a nation-wide longitudinal study. Cardiovasc Diabetol 15:1

15. Ou HT, Chang KC, Li CY, Wu JS (2017) Comparative cardiovascular risks of dipeptidyl peptidase 4 inhibitors with other secondand third-line antidiabetic drugs in patients with type 2 diabetes. $\mathrm{Br}$ J Clin Pharmacol 83:1556-1570

16. Hsu CC, Wahlqvist ML, Lee MS, Tsai HN (2011) Incidence of dementia is increased in type 2 diabetes and reduced by the use of sulfonylureas and metformin. J Alzheimers Dis 24:485-493

17. Biessels GJ, Staekenborg S, Brunner E, Brayne C, Scheltens P (2006) Risk of dementia in diabetes mellitus: a systematic review. Lancet Neurol 5:64-74

18. Heckman J, Ichimura H, Smith J, Todd P (1998) Characterizing selection bias using experimental data. Econometrica 66: 10171098

19. Dehejia RH, Wahba S (2002) Propensity score-matching methods for nonexperimental causal studies. Rev Econ Stat 84:151-161

20. Charlson M, Szatrowski TP, Peterson J, Gold J (1994) Validation of a combined comorbidity index. J Clin Epidemiol 47:1245-1251
21. Chang HY, Weiner JP, Richards TM, Bleich SN, Segal JB (2012) Validating the adapted diabetes complications severity index in claims data. Am J Manag Care 18:721-726

22. Hosmer DW Jr, Lemeshow S, May S (2008) Applied survival analysis: regression modeling of time to event data, 2 nd edn. Wiley, Chichester

23. Chang CH, Jiang YD, Chung CH, Ho LT, Chuang LM (2012) National trends in anti-diabetic treatment in Taiwan, 2000-2009. J Formos Med Assoc 111:617-624

24. Nissen SE, Wolski K (2007) Effect of rosiglitazone on the risk of myocardial infarction and death from cardiovascular causes. N Engl J Med 356:2457-2471

25. Rosen CJ (2010) Revisiting the rosiglitazone story-lessons learned. N Engl J Med 363:803-806

26. Heneka MT, Landreth GE (2007) PPARs in the brain. Biochim Biophys Acta 1771:1031-1045

27. Heneka MT, Reyes-Irisarri E, Hull M, P Kummer M (2011) Impact and therapeutic potential of PPARs in Alzheimer's disease. Curr Neuropharmacol 9:643-650

28. Sharma B, Singh N (2011) Behavioral and biochemical investigations to explore pharmacological potential of PPAR-gamma agonists in vascular dementia of diabetic rats. Pharmacol Biochem Behav 100:320-329

29. Kaur B, Singh N, Jaggi AS (2009) Exploring mechanism of pioglitazone-induced memory restorative effect in experimental dementia. Fundam Clin Pharmacol 23:557-566

30. Kumar P, Kaundal RK, More S, Sharma SS (2009) Beneficial effects of pioglitazone on cognitive impairment in MPTP model of Parkinson's disease. Behav Brain Res 197:398-403

31. Hanyu H, Sato T, Kiuchi A, Sakurai H, Iwamoto T (2009) Pioglitazone improved cognition in a pilot study on patients with Alzheimer's disease and mild cognitive impairment with diabetes mellitus. J Am Geriatr Soc 57:177-179

32. Sato T, Hanyu H, Hirao K, Kanetaka H, Sakurai H, Iwamoto T (2011) Efficacy of PPAR- $\gamma$ agonist pioglitazone in mild Alzheimer disease. Neurobiol Aging 32:1626-1633

33. Wang KC, Woung LC, Tsai MT, Liu CC, Su YH, Li CY (2012) Risk of Alzheimer's disease in relation to diabetes: a populationbased cohort study. Neuroepidemiology 38:237-244

34. Shen HN, Lu CL, Li CY (2012) Dementia increases the risks of acute organ dysfunction, severe sepsis and mortality in hospitalized older patients: a national population-based study. PLoS One 7: e42751

35. Liao KM, Ho CH, Ko SC, Li CY (2015) Increased risk of dementia in patients with chronic obstructive pulmonary disease. Medicine (Baltimore) 94:e930 